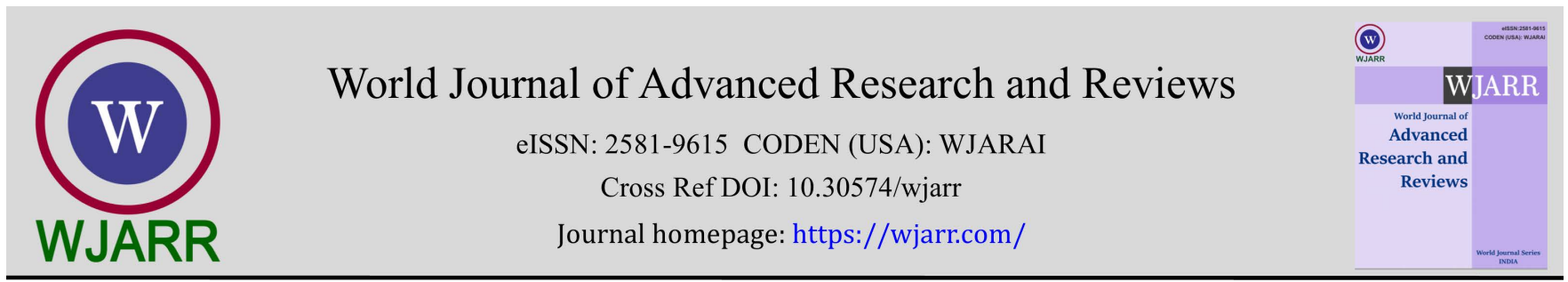

(REVIEW ARTICLE)

Check for updates

\title{
The contribution of vaccination to global health, from the past to the present: mandatory or social duty?
}

Barbara Fialho Carvalho Sampaio 1, 2,3, ${ }^{*}$, Leandro Gregorut Lima 1,3, Marco Aurélio Silvério Neves 1,3, Carolina Paulette Neves ${ }^{3}$ and Heitor Franco de Andrade Júnior ${ }^{1,2}$

${ }^{1}$ School of Medicine of the University of São Paulo - Department of Pathology and Experimental Pathophysiology - Brazil.

${ }^{2}$ Institute of Tropical Medicine of the University of São Paulo - Department of Protozoology-Brazil.

${ }^{3}$ Arthron Medical Services - Scientific Department-Brazil.

World Journal of Advanced Research and Reviews, 2022, 13(01), 058-064

Publication history: Received on 16 November 2021; revised on 22 December 2021; accepted on 24 December 2021

Article DOI: https://doi.org/10.30574/wjarr.2022.13.1.0714

\begin{abstract}
Vaccines have saved millions of lives and reduced the severity of many infections, but today the reduction in vaccine coverage has been reflected in the resurgence of epidemics of mumps, whooping cough, measles and chickenpox. This has happened because many people do not recognize the effectiveness of vaccination and fear the side effects, in other words, the main concern is the safety of the vaccines. As a consequence, the greater responsibility of the individual and respect for his will can lead authorities to less vigorously promote the "duty of vaccination", which is also a social duty. Unfortunately, however, the attitude of individuals is guided by their beliefs about health, which are often supported by an erroneous perception of risk arising from false news.
\end{abstract}

Keywords: Vaccine; Epidemic; Pandemic; Social impact; Fake News

\section{Introduction}

Vaccines have a history that begins at the end of the $18^{\text {th }}$ century, but it was only from the end of the $19^{\text {th }}$ century that they could be developed in the laboratory[1].

The impact of vaccines on longevity and human health is indisputable. The use of safe and effective vaccines is a wellestablished and cost-effective public health intervention in the last three decades, in addition to having a great impact on the prevalence of infectious diseases. Each year, they prevent more than three million deaths, successfully consolidating themselves in the prevention of infectious diseases ${ }^{[1]}$.

The initiation of vaccination, defined as an overt attempt to use part or all of a microbial pathogen to protect against this pathogen, likely originated in homeopathic beliefs about small doses of diseases that protect against serious diseases, empirically verified by ingesting small doses of poison to prevent the intentional fatal poisoning of rulers by rivals. By the $11^{\text {th }}$ century, there was evidence in the Chinese literature of the use of inflated smallpox scabs on the nose to immunize against smallpox, perhaps based on observations that previous smallpox protected against subsequent exposure [2,3].

Although variolation was a success (for example, as confirmed during the American Revolution by the immunity of British troops from smallpox outbreaks and Washington's later decision to inoculate its army), significant and even fatal reactions acted as a deterrent to its use. No doubt this was the impetus for Jenner's historical observation that cowpox,

\footnotetext{
${ }^{*}$ Corresponding author: Barbara Fialho Carvalho Sampaio

School of Medicine of the University of São Paulo - Department of Pathology, and Experimental Pathophysiology - Brazil. 
a mild disease in humans, could prevent smallpox. This discovery not only led to the eradication of smallpox in the 20th century, but also highlighted the idea of deliberate protection against exposure to infectious diseases [4].

The history of vaccination as a deliberate effort began with Louis Pasteur. The scientist was on vacation in the summer of 1881 and returned in the fall to study chicken cholera, caused by what we now call Pasteurella multocida. A culture left on the bench over the summer was inoculated into chickens but did not cause disease. Pasteur then made a new culture and inoculated the same chickens, either for parsimony or purpose, a fact that we do not know. Anyway, the chickens were resistant to the new challenge, which made the scientist realize that the aged culture had made them immune ${ }^{[5]}$.

From these observations, Pasteur built the hypothesis that pathogens could be attenuated by exposure to environmental aggressions, such as high temperature, oxygen and chemicals. Their subsequent work on anthrax and rabies confirmed hypothesis [6]. In the next century, Calmette and Guérin used the artificial medium passage to attenuate Mycobacterium bovis ${ }^{[7]}$, and Theiler used the passage in mouse and chicken embryos to attenuate the fever virus yellow ${ }^{[7]}$.

Meanwhile, the concept of antibodies and cellular immune responses was developed from the original work of Paul Ehrlich and Ilya Metchnikoff, respectively, and their measurement by relatively primitive methods established the dual nature of the adaptive immune system. Consequently, vaccinologists focused on stimulating these responses ${ }^{[8]}$.

Despite this progress, vaccine-preventable diseases remain a major cause of morbidity and mortality. In addition, adoption of new vaccines by low- and middle-income countries has been slower than in richer countries.

According to State of the World's Vaccines and Immunization report [9] "Vaccination - even with the addition of new, more expensive vaccines - remains one of the most cost-effective health interventions." The publication notes that more boys and girls are reached with immunization, about a hundred million children a year in the period 2005-2007, approximately. And the benefits of immunization are increasingly extended to adolescents and adults, providing protection against potentially fatal diseases, such as the flu, meningitis and cancers that occur in adulthood ${ }^{[10] .}$

The vision for the "Vaccine Decade (2011-2020)" was of a world where all individuals and communities could enjoy a life free of vaccine-preventable diseases. This mission was the central objective to expand, by 2020, the full benefit of immunization to all people, regardless of where they are born, live or who they are. This vision of a world without vaccine-protected diseases becomes a utopia, but even so, we have to try to achieve the unattainable.

There is a growing demand for vaccine safety, fueled in part by anti-vaccination groups. As the disease recedes, the need for vaccination becomes less evident to the public and more people choose not to sign the social contract to be vaccinated, depending instead on the herd immunity of the vaccinated people around them. Of course, mass immunity will fail if too many refuse to be vaccinated. However, there is another important point in this scenario: there are real safety problems associated with vaccines, such as paralysis after oral polio vaccine and disseminated infections after Bacille Calmette-Guérin [11,12,13,14].

For this reason, older vaccines need to be re-examined to see if safety can be improved, as was done by replacing the whole cell pertussis vaccine with acellular pertussis vaccine and replacing the rabies vaccine made in the brain with vaccine made in cell culture.

In fact, one of the advantages of new molecular technologies is improved security. As the risk-benefit ratios become more controversial as the presence of the disease decreases, it will be important to minimize vaccine-associated reactions. On the other hand, zero risk is impossible to achieve, and there will always be tension between public health needs and the regulatory push to protect against even remote and theoretical risks. This last trend acts as a brake on the rapid application of new public health measures. Thus, there is disagreement as to whether to err on the side of safety or disease prevention.

By the 1960s, the vast majority of deaths and serious illnesses attributable to common childhood infectious diseases and preventable by vaccination were occurring in children in the developing world, where coverage with vaccines such as measles was often less than $5 \%$ and mostly restricted. To children. In the early 1960 s, about a third of African children were under the age of 5 , and infectious diseases, particularly measles, were responsible for a substantial proportion of these deaths ${ }^{[12,13,14] .}$.

Faced with this challenge, the World Health Organization (WHO) established the Vaccination Program in 1974 to increase the intake of routine childhood vaccines worldwide. This program has been very successful, with Vaccination 
Program vaccine coverage rates skyrocketing from less than $5 \%$ to over $80 \%$ in many low- and lower-middle-income countries[15]. In the 1980s, coverage with Vaccination Program vaccines in many low-income settings was similar or even better than that achieved in many parts of the industrialized world, where childhood infectious diseases were no longer seen as a significant threat.

The success of the Vaccination Program was achieved in part because of strong leadership at WHO in many developing countries, and in part through financial support from the international community. Because Vaccination Program vaccines are relatively inexpensive when mass produced, a complete immunization of a child cost about $\$ 15$ in the 1990s. The introduction of effective national programs in most developing countries has led to large reductions in deaths and hospital admissions for measles and neonatal tetanus ${ }^{[15] .}$

It is estimated that in 2012 there were around 157,000 deaths from measles, a dramatic decrease from the situation 20 years ago, but still with an unacceptable burden of preventable infection. There has also been a dramatic reduction in the number of neonatal tetanus deaths (over 90\% since the 1980s), achieved through the routine immunization of mothers attending antenatal clinics with tetanus toxoid, but it is estimated that there was still an estimated 60,000 preventable deaths from this infection in 2012. Indeed, the impact of vaccination was not limited to the developing world and a recent review from the United States estimated that 103 million cases of selected infectious diseases were prevented by vaccination since 1924 [16].

Although coverage with the starter pack of routine childhood vaccines (BCG, DPT, measles and polio) is now as high in many low- and middle-income countries as it is in the industrialized world, many more lives could be saved by reaching underserved populations with vaccines. Of the Vaccination Program, such as measles and influenza. Finding better ways to reach these groups, who are responsible for an increasing proportion of child morbidity and mortality in many developing countries, with appropriate health interventions, is likely to be an important goal of the post-2015 development goals. Methods being explored include gaining a better understanding of why these communities or individuals are resistant to vaccination, making it more accessible through home visits and using technologies to disseminate quality scientific knowledge, bringing safety to the population to get vaccinated [17].

Sensitive handling of the issue of vaccine safety is a key factor in ensuring high vaccine uptake. All vaccines have side effects in a small proportion of vaccine recipients and this needs to be recognized while emphasizing the benefits that come from vaccination. Recently developed rotavirus vaccines provide a good example of this principle. Both widely used rotavirus vaccines cause the severe condition of intussusception in a small proportion of vaccine recipients, perhaps one to five additional episodes per 100,000 vaccines, an acceptable risk considering the large reduction in hospital admissions and deaths achieved with these vaccines [18]. The issue of true vaccine side effects needs to be separated from incorrect claims, such as the reported association between the MMR vaccine and autism, and these false claims vigorously refuted [19].

As vaccines are fundamental tools for the maintenance of public health, governments have an important role in their dissemination through recommendations and purchases. Furthermore, it has become obvious that governments must be proactive in preventing vaccine shortages through multi-vendor incentives.

Lack of information and false news are recognized as one of the main factors contributing to low vaccination coverage. In particular, the increased risk of vaccine-preventable disease outbreaks and reduced vaccine coverage may be related to vaccine hesitancy [20].

Anti-vaccination campaigns had a detrimental impact on vaccine uptake. For example, the link between the mumpsmeasles-rubella vaccine and autism described in a now-reported article published by The Lancet in 1998 that questioned the safety of vaccines ${ }^{[20,21]}$.

Therefore, the application of mandatory vaccination is one of the strategies that some countries are adopting and others are considering facing this issue of anti-vaccination groups. After all, policies requiring vaccines have always been controversial and related to opposition and dispute. But in fact something needs to be done to change this scenario, after all, according to a recent American survey, more than $10 \%$ of parents refused mandatory vaccinations and objected to their safety and usefulness ${ }^{[22] .}$

National and regional immunization policies are subsidized by vaccination coverage. In particular, it can influence interventions performed and increase or decrease vaccine absorption [23]. 
Furthermore, with regard to hepatitis B, tetanus, diphtheria, whooping cough, Haemophilus influenza type B, hepatitis B, poliovirus, measles, mumps and rubella, in 2016 about two-thirds of Italian regions had coverage rates below $90 \%$ [24]. In recent years, a significant decline in childhood vaccination coverage rates has remained an almost worldwide pattern. As a result, due to inadequate vaccination coverage, infectious diseases continue to occur, increasing mortality and morbidities that could be prevented by the act of getting vaccinated [25].

Considering, for example, measles outbreaks and the European scenario, recent epidemics have occurred in Romania (7570 cases), Italy (5006 cases), Ukraine (4667) and Germany (891 cases). It is noteworthy that $87 \%$ of cases occurred in unvaccinated people ${ }^{[26]}$.

These outbreaks have led to a discussion about vaccination policies in any European country. All countries in the European Union have a long tradition of vaccination programs, but there is still much that can be done to improve and accelerate vaccination coverage. In this European setting, there are big differences between countries considering the type of vaccine used, number of doses and time of vaccination, but also differences in whether vaccines are recommended or mandatory.

In eleven of thirty-one countries, there is at least one vaccine mandatory to increase immunization coverage. There are many reasons why European countries are still not meeting their collective immunization needs. In particular, the lack of understanding and misperceptions of vaccination by the public and sometimes by health professionals and misinformation circulating in the mass media have undermined parental confidence in vaccines [27].

In contrast to vaccination policies, anti-vaccination campaigns contribute to the spread of false news. People who have been asked to vaccinate their children are often frightened by such erroneous information, harming the population's adherence to the collective vaccination system.

While it is preferable that high demand and community acceptance make mandatory vaccination programs unnecessary, the World Health Organization (WHO) understands that some countries may want to move in this direction when faced with declining vaccination rates and disease outbreaks [28].

Resurgent outbreaks of vaccine-preventable diseases that have been controlled or eliminated have been observed in many contexts, as seen in recent epidemics and pandemics could be prevented through vaccination. This is because vaccination campaigns can prevent or even control outbreaks, but they must be worked out with considerable certainty, and real-time surveillance can provide valuable information about the population at risk and what the main vaccination targets for blockade would be. Of possible outbreaks. However, we run into preventive diagnostic limitations, since the ideal would be to confirm the immunological status of each individual, before an unnecessary revaccination [29].

It is important to recognize the possibility of recurrent outbreaks, epidemics and pandemics in this post-elimination phase and/or increased population immunity through vaccination, with the need to prepare a constant, sensitive and interconnected surveillance system between continents to block the beginning outbreaks, acting in a preventive and assertive manner. Available vaccines are effective, but the protection they evoke diminishes over time. The use of booster doses can control flare-ups, but it is not precisely defined whether they can prevent them. The rapid decline in antibody levels may limit the impact of introducing a third dose into the recommended immunization schedule.

The real reasons for the development of outbreaks in people with very high vaccination coverage are not clearly understood. The use of a booster dose or the preparation of vaccines containing the emerging serotypes are possible solutions. Studies primarily dedicated to improving our knowledge of the immune response to vaccines are needed so that those effective against long-term disease can be prepared and outbreaks can be avoided [30].

Epidemiological studies conducted in the pre-vaccination era have led to the conclusion that a natural wild-type infection typically confers lifelong protection against the viruses, although cases of reinfection have been reported. Resurgent outbreaks of infectious diseases, such as in the case of mumps and measles, cannot be treated as a problem that will only affect children, and should be a warning sign for the older population, due to the decay time of vaccine antibodies from protection $[31,32]$.

Thus, epidemiological and vaccine control studies, through non-invasive immunological tests, can be used as adequate public health measures, such as revaccination, which can be adequately planned.

The traditional definition of vaccination doubt coined by the WHO, which in a very general way considers it a "delay in the acceptance of vaccination or rejection of vaccines, despite the availability of vaccination services" [33] was recently 
reformulated by the European Union Commission which, in 2018 , defined it as a form of "distrust in science and fear of possible side effects" due to a series of factors, including "lack of reliable information and, in some cases, distrust in the providers of available information " [34].

Consequently, it is easy to intuit that not all refusals to undergo prophylactic practices by citizens can be redirected to the phenomenon of vaccination doubt [35]. In fact, recognized doctrine has highlighted that there are basically some causes that can lead the population to not submitting to State-recommended vaccines: strict medical reasons, religious reasons, philosophical reasons for life and lack of confidence in vaccination programs [36] and, specifically, in the effect of vaccines on the health of the recipient.

It is well known that these prophylactic techniques can be very dangerous for the immunosuppressed, that is, those individuals who, affected by a wide spectrum of immunological disorders, present serious abnormalities in the functioning of the immune system and, therefore, cannot be vaccinated [37]. No there is no doubt that this group of individuals cannot be related in any way to vaccine hesitation, since the decision not to be vaccinated is due solely to the health status of its components. Within the population there is also a group of people who are convinced that interfering with the normal process of human health is contrary to their religious beliefs or philosophy of life [38]. Although this position has some controversial aspects and it is necessary to take into account its consequences, it does not it seems possible to redirect it to the field of vaccine doubt because those who take it do not show any skepticism, in terms of health, about the secondary effects of these specific preventive practices, as they only express the intention to live according to their own convictions. Spiritual [39].

As already highlighted, underestimating the phenomenon of vaccination doubt entails serious social costs that would have been avoided if the problem had been resolved in advance. As shown, for example, the Spanish situation, characterized by a very high percentage of vaccinated [40], in which there is no coercive measure, so that to obtain high rates of vaccination coverage it is not necessary to resort to strict sanction measures, on the contrary, they could have a negative impact, as pointed out in a study by a WHO-funded working group ${ }^{[41]}$ and pertinent doctrine that prefers to consider respect for the vaccination program as a civic duty rather than a strictly legal one. Paradoxically, it is the same statistical history of countries that show that it is not essential to introduce negative sanctions to obtain a level of vaccine coverage compatible with the percentages necessary to achieve group immunity ${ }^{[42]}$.

Therefore, the process of immunization of society to anti-vaccination movements is nothing more than measures with attempts to nullify science, which today lacks special legal protection due to the loss of credibility and the dissemination of false news. In short, due to the effect of false news added at the time to the peculiarities of COVID-19, it is clear that the pandemic scenario makes it difficult for the population to accept vaccines produced in record times, even with their published efficacy and safety rates and Authorized by world regulatory agencies [43]. For this reason, preventing antivaccination movements from feeding on false news is an absolute necessity.

With pedagogical scientific work, broadly communicative, that values and the development of solutions based on science in the face of the sanitary crisis that plagues us, it will be possible to prevent the so-called vaccine doubt, thus producing greater efficiency in communication, encouraging the use of vaccines already marketed for infectious diseases, especially in the current scenario of COVID-19. Thus, it is easy to reduce the doubt about whether to vaccinate or not, reducing the impact of false news about vaccines.

\section{Conclusion}

It is highly insufficient to base COVID-19 vaccine promotion campaigns solely on information provided by health and national authorities. Additional expert activities need to be presented and employ social and traditional online media to provide accurate but understandable information to non-specialists covering the vaccines mechanism of action, the research process, approval regulations, and individual and public benefits of COVID vaccinations -19 , as well as your security profile.

To ensure visibility and high quality, all these initiatives need to be supported by organized groups of high-ranking independent experts, without conflicts of interest and with the professional support of experienced public relations agencies and local media. Partnering with public institutions such as universities, libraries and schools is also highly recommended as they are a valuable source through which evidence-based information can be communicated to the public. It is recommended that these expert groups cover a wide range of scientific fields as well as public health to develop more effective strategies to increase adherence to vaccination. 


\section{Compliance with ethical standards}

\section{Acknowledgments}

I thank Professor Dr. Heitor Franco de Andrade Júnior for his encouragement and support in my research on vaccines and populations. We appreciate the support of all departments involved, School of Medicine of the University of São Paulo - Department of Pathology and Experimental Pathophysiology - Brazil; Institute of Tropical Medicine of the University of São Paulo - Department of Protozoology- Brazil and Arthron Medical Services - Scientific DepartmentBrazil.

\section{Disclosure of conflict of interest}

The authors declare that there are no conflicts of interest in the preparation of this manuscript.

\section{Statement of ethical approval}

This document is a clinical research protocol that will be conducted in compliance with all stipulations of this protocol, current national regulations, and the guidelines established by the Document of the Americas and the ICH Good Clinical Practice Guide.

\section{References}

[1] Ehreth J. The value of vaccination: a global prespective. Vaccine. 2003; 21(27-30): 4105-17.

[2] Fenner F, Henderson DA, Arita I, Jezek Z, Ladnyi ID. History of International Public Health, No. 6.1988. Early efforts at control: variolation, vaccination, and isolation and quarantine. 245-276.

[3] Plotkin SL, Plotkin SA. Vaccines. A short history of vaccination. 2004; 1-15.

[4] Pasteur L. De l'attenuation du virus du cholera des poules. C. R. Acad. Sci. Paris. 1880; 91: 673-680.

[5] Pasteur L, Chamberland C-E. Sur la vaccination charbonneuse. C. R. Acad. Sci. Paris. 1881; 92: 1378-1383.

[6] Calmette A, Guerin C, Breton M. Contribution a l'etude de la tuberculose experimental du cobaye (infection et essais de vaccination par la voie digestive) Ann. Inst. Pasteur Paris. 1907; 21: 401-416.

[7] Theiler M, Smith HH. The use of yellow fever virus by in vitro cultivation for human immunization. J. Ex Med. 1937; 65: 787-800.

[8] Plotkin SA. Vaccines: past, present and future. Nat Med. Apr 2005; 11(4): S5-11.

[9] World Health Organization, UNICEF, World Bank. State of the world's vaccines and immunization. 3rd ed. Geneva: WHO; 2009.

[10] World Health Organization. Global Vaccine Action Plan 2011 - 2020

[11] McCurdy LH, Larkin BD, Martin JE, Graham BS. Modified vaccinia Ankara: potential as an alternative smallpox vaccine. Clin. Infect. Dis. 2004; 38: 1749-1753.

[12] WHO collaborative study group: The relationship between persisting spinal paralysis and poliomyelitis vaccine-results of a ten-year enquiry. Bull WHO60. 1982; 231-242.

[13] Hoft DF, et al. Clinical reactogenicity of intradermal bacille Calmette-Guerin vaccination. Clin. Infect. Dis. 1999; 28: 785-790.

[14] Horwitz MA, Harth G. A new vaccine against tuberculosis affords greater survival after challenge than the current vaccine in the guinea pig model of pulmonary tuberculosis. Infect. Immun. 2003; 71: 1672-1679.

[15] World Health Organisation. Global routine vaccination coverage 2012. Wkly Epidemiol. Rec. 2013; 88, 482-485.

[16] Van Panhuis WG, et al. Contagious diseases in the United States from 1888 to the present. N. Engl. J. Med. 2013; 369: 2152-2158.

[17] Arora NK, Lal AA, Hombach JM, Santos JI, Bhutta ZA, Sow SO, Greenwood B. The need for targeted implementation research to improve coverage of basic vaccines and introduction of new vaccines. Vaccine. 2013; 31S: B129B136. 
[18] Glass RI\& Parashar UD. Rotavirus vaccines-balancing intussusception risks and health benefits. N. Engl. J. Med. 2014; 370: 568-570.

[19] Wakefield AJ, et al. Ileal-lymphoid-nodular hyperplasia, non-specific colitis, and pervasive developmental disorder in children. .Lancet. 1998; 351: 637-641.

[20] Allen A. Vaccine-the controversial story of medicine's greatest lifesaver. New York, NY: W.W. Norton \& Co. 2007.

[21] Weber T. Alfred Russel Wallace and the antivaccination movement in Victorian England. Emerg. Infect. Dis. 2010; 16: $664-668$.

[22] Henderson DA. Smallpox—the death of a disease. Amherst, NY: Prometheus Books. 2009.

[23] Geison GL. The private science of Louis Pasteur. Princeton, NJ: Princeton University Press. 1995.

[24] Wu X, Smith TG Rupprecht CE. From brain passage to cell adaptation: the road of human rabies vaccine development. Expert Rev. Vac. 2011; 10: 1597-1608.

[25] Burioni R, Odone Am, Signorelli C. Lessons from Italy's policy shift on immunization. Nature, Correspondence, February. 2018.

[26] Coombes R. Steps up action against vaccine hesitancy as measles outbreaks continue. BMJ. $2017 ; 359$ : j4803.

[27] Schmitt HJ, Booy R, Weil-Olivier C, Van Damme P, Cohen R, Peltola H. Child vaccination policies in Europe: a report from the summits of independent European vaccination experts. The Lancet Infectious Disease. 2003; 3(2): 1038.

[28] Walkinshaw E. Mandatory vaccinations: the international landscape. CMAJ. 2011; 183(16): E1167-8.

[29] Beleni AI, Borgmann S. Mumps in the Vaccination Age: Global Epidemiology and the Situation in Germany. Int J Environ Res Public Health. Jul 2018; 31; 15(8): 1618.

[30] Principi N, Esposito S. Mumps outbreaks: A problem in need of solutions. J Infect. 2018; 76(6): 503-6.

[31] Nageswaran P, Jenner L, Paul S Resurgence of measles and mumps: not just a childhood problem. Br J Nurs. 2017; 26(8): 471.

[32] Vandermeulen C, Roelants M, Vermoere M, Roseeuw K, Goubau P, Hoppenbrouwers K. Outbreak of mumps in a vaccinated child population: a question of vaccine failure? Vaccine. 2004; 22(21-22): 2713-6.

[33] SAGE Working Group on Vaccine Hesitancy, WHO. Re- port on Vaccine Hesitancy. 2015: 1.

[34] Comisión Europea. COM (2018) 244/2 2018/0115 (NLE). 0 cit.: 1, 2.

[35] Di Pietro ML, Refolo, y González Melado F. « On the "responsibility” of vaccination ». Bioethics Notebooks, 2012, XXIII, 78: 323-340.

[36] Bellver Capella, V. « Vaccines. Right and ... obligation? ». ROL. Revista de Enfermería. 2015; 38(10): 664.

[37] Pertusa Martínez, S. «Immunosuppressed patients: definition and special precautions ». FMC. Continuing Medical Training in Primary Care. 2019; 26(10): 548-562.

[38] Bellver Capella, V. « Vaccines. Right and ... obligation? ». 0 cit. 38 (10): 664.

[39] For thematic and extension reasons we cannot deal with this specific form of resistance to vaccination, which would open up reflections on conscientious objection. For an analysis in this regard, it is forwarded to Biondo, F. «Obiezione di coscienza e vulnerabilità. The dark side of the movement of resistance to compulsory vaccinations ". Ragion Pratica. 2019; 52(1): 169-183.

[40] The primary vaccination campaigns of the Ministry of Health have transformed Spain into a leading country in the world in terms of the number of vaccinated children, which, in 2018, exceeded $98 \%$ for poliomyelitis. Ministry of Health, 2020.

[41] AGE Working Group on Vaccine Hesitancy, WHO. Report on Vaccine Hesitancy. 2014; 45.

[42] Bellver Capella V. «Vaccines. Right and ... obligation?». 0 cit. 38(10): 667.

[43] As shown by the data produced in the barometer published by the Spanish Sociological Research Center (CIS), $43.8 \%$ of Spaniards would reject the application of the vaccine (CIS, «Barometer of October. , study number 3926», 2020. 